\title{
Features and models for human activity recognition
}

\author{
Silvia González ${ }^{\mathrm{a}}$, Javier Sedano ${ }^{\mathrm{a}}$, José R. Villar ${ }^{\mathrm{b}}$, Emilio Corchado ${ }^{\mathrm{c}}$, Álvaro Herrero ${ }^{\mathrm{d}, *}$, \\ Bruno Baruque $^{\mathrm{d}}$ \\ a Instituto Tecnológico de Castilla y León, Burgos, Spain \\ ${ }^{\mathrm{b}}$ University of Oviedo, Campus de Viesques s/n 33204 Gijón, Spain \\ c Departamento de Informática y Automática, University of Salamanca, Spain \\ ${ }^{\mathrm{d}}$ Department of Civil Engineering, University of Burgos, Spain
}

\section{A R T I C L E I N F O}

\section{Article history:}

Received 19 March 2014

Received in revised form

28 November 2014

Accepted 26 January 2015

Available online 8 May 2015

\section{Keywords:}

Human activity recognition

Genetic fuzzy finite state machine

Feature domain reduction

Feature selection

Information correlation coefficient

\begin{abstract}
A B S T R A C T
Human Activity Recognition (HAR) is aimed at identifying current subject task performed by a person as a result of analyzing data from wearable sensors. HAR is a very challenging task that has been applied in different areas such as rehabilitation and localization. During the past ten years, plenty of models, number of sensors and sensor placements, and feature transformations have been reported for this task. From this bunch of previous ideas, what seems to be clear is that the very specific applications drive to the selection of the best choices for each case.

Present research is focused on early diagnosis of stroke, what involves reducing the feature space of gathered data and subsequent HAR, among other tasks. In this study, an Information Correlation Coefficient (ICC) analysis was carried out followed by a wrapper Feature Selection (FS) method on the reduced input space. Additionally, a novel HAR method is proposed for this specific problem of stroke early diagnosing, comprising an adaptation of the well-known Genetic Fuzzy Finite State Machine (GFFSM) method.

To the best of the author's knowledge, this is the very first analysis of the feature space concerning all the previously published feature transformations on raw acceleration data. The main contributions of this study are the optimization of the sample rate, selection of the best feature subset, and learning of a suitable HAR method based on GFFSM to be applied to the HAR problem.
\end{abstract}

c 2015 Elsevier B.V. All rights reserved.

\section{Introduction}

This research aims at developing a solution for the early diagnosis of stroke and the rehabilitation of elder people after a disruptive event: an injury due to a falling, a seizure onset, etc. In this context, only a small subset of activities are to be identified among those that a human being can usually do and hence, the recognition of those activities is simplified. On the other hand, activity recognition devices help to improve the mobility of elder people during rehabilitation, so technology is enhancing the quality of life for both elder and injured people.

Every human being performs different activities during the day and Human Activity Recognition (HAR) targets their identification. Though walking recognition is nowadays a clear-cut task [1], the recognition of other activities is not. It is difficult due to the fact that there are many different activities that a person may perform and some of them could even co-occur at the same time (talking to another person or eating a sandwich while walking, reading and

\footnotetext{
* Corresponding author.
}

being seated). Additionally, a wide spread of feature transformations and HAR methods have been applied up to now [2-4]. Many techniques of data gathering, including video-images, are being used but tridimensional accelerometers are the data sources for the majority of previous HAR studies.

The main problem to be solved in this research is the early diagnosis of stroke onsets. During such episodes, the upper limbs are the parts of the body that best reflect the differences regarding normal behavior. According to this idea, two triaxial accelerometers are usually placed on the subject's wrists. The hypothesis is that with these sensors we would be able to recognize an onset due to the differences in the movement patterns. However, these movement patterns will depend on the task that the subject is carrying out. Therefore, early diagnosis of stroke also includes HAR. Interesting enough to mention, the activities to be identified also depends on the focused population; generally speaking, the older you are the lower the amount of activities you perform during everyday life. Additionally, the quality of the movement may be rather different depending on the age of people; the younger the faster. Thus, the target population define the different 
activities that may be excluded for recognition and the age reduces the amount of movement; movement for the elderly is lower than that for younger people while performing the same activities.

A light wearable device might be enough for the data gathering, pre-processing and analyzing provided the number of activities to be recognize is kept small. Previous studies have analyzed the window size for pre-processing the continuous data flow coming from data sources [5]. Once the data is properly pre-processed, they must be analyzed.

To do so, one of the most interesting HAR techniques that can be deployed in embedded devices is the Genetic Fuzzy Finite State Machine (GFFSM) [6], which also handles expert knowledge with high accuracy. To this end, this study is focused on enhancing the HAR by using 3DACC sensors in wearable devices. The aim of this research is two-fold: on the one hand, the selection of the 3DACC transformations is analyzed and a feature subset is chosen; on the other hand, the improvements on the GFFSM model obtained due to the reduced feature subset are explored. The study also makes use of this GFFSM model for HAR, but additionally it tackles several issues identified as relevant in the HAR literature, such as those concerning the diversity of transformations from the acceleration raw data and how to reduce the dimensionality and the different methods for cross validation used so far.

The remainder of the present paper is organized as follows. Next section deals with the challenging task of HAR, including an overview of the input feature domain, as well as an in-depth review of the HAR literature. Section 3 introduces the proposed method and its different stages: a two-step Feature Selection (FS) and a HAR modeling by means of the GFFSM. Subsequently, Section 4 is devoted to evaluate and discuss the experimentation carried out. Finally the main conclusions from the obtained results as well as the future work are drawn in Section 5.

\section{A review of human activity recognition}

After several years of study, a wide spectrum of features, calculated as transformations from raw acceleration data, have been proposed for HAR. A set of features is chosen for each one of the applied methods according to different criteria. This section describes the main features from those that have been proposed in the literature up to now; afterwards, the previous work on HAR is analyzed and compared.

\subsection{From acceleration data to the input feature space}

Nowadays, the most common sensor applied in HAR is the triaxial accelerometer. Data gathered from this type of sensor, known as raw data (RD, $a_{i}^{x}, a_{i}^{y}$ and $a_{i}^{z} ; a_{i, j \in\{x, y, z\}}$ for the sake of brevity), should be decomposed in the gravity acceleration $(G)$ that is due to each gravity, $g_{i}^{x}, g_{i}^{y}$ and $g_{i}^{z}$ or $g_{i, j \in x, y, z^{-}}$and the BA which is due to the human movement, $b_{i}^{x}, b_{i}^{y}$ and $b_{i}^{z}$ or $b_{i, j \in x, y, z^{-}}$. The ability of BA to discriminate among different human gestures is documented in [7]. Nevertheless, the literature includes the use of a wide variety of transformations (the most interesting ones are described below), where $w$ stands for the window size - if needed-, and sub-indexes $i \in\{1, \ldots, N\}$ and $j \in\{x, y, z\}$ stand for the number of the sample and the axis, respectively. It is worth mentioning that all these features computed on each one of the possible signals ( $R D, B A$, and $G$ ) would generate a feature space with more than 190 features, whose processing and analysis are very challenging tasks indeed.

The following features have been previously applied to HAR:

1. The mean, deviation and higher momentum statistics values for the RD [8] or for the BA [9,7], and the RD mean absolute deviation $M A D_{j}=\frac{1}{w} \sum_{i=1}^{w}\left|a_{i, j}-m_{j}\right|[10,8]$, where $m_{j}$ is the mean value of $a_{i, j}$.

2. The Root Mean Square $R M S_{j}=\sqrt{\frac{1}{w} \sum_{i=1}^{w}\left|a_{i, j}^{2}\right|}[10]$.

3. The sum of the absolute values of the BA [11] $s B A_{i}=$ $\frac{1}{w} \sum_{t=i}^{i+w} \sum_{j \in\{x, y, z\}}\left|b_{t, j}\right|$, the vibration of the sensor $(\Delta)$ [9] $\Delta_{i}=\frac{1}{w} \sum_{t=i}^{i+w} \sum_{j \in\{x, y, z\}} a_{t, j}^{2}-g_{t, j}^{2}$ and the tilt of the body ( tilt $_{i}=\frac{1}{w} \sum_{t=i}^{i+w}\left|a_{i}^{y}\right|+\left|a_{i}^{z}\right|[6]$. The two former transformations were designed to detect whether the sensor registers no movement at all, as fixed to an steady object, while the latter is used whenever the sensor axes match with the body axes.

4. The Signal Magnitude Area $S M A=\frac{1}{w} \cdot \sum_{i=1}^{w}\left(\left|b_{i}^{x}\right|+\left|b_{i}^{y}\right|+\left|b_{i}^{z}\right|\right)$ $[9,12,7]$ discriminating between gravity acceleration and BA.

5. The Amount of Movement $A M_{i}=\sum_{v=\{x, y, z\}} \mid \max _{t=i+1}^{i+w}\left(b_{t}^{v}\right)-$ $\min _{t=i+1}^{i+w}\left(b_{t}^{v}\right) \mid$ [6] calculated as the maximum difference between the values of BA within the sliding window.

6. The Delta coefficients for estimating the first order time derivative of each of the $G$ signal components [12]: $\Delta g_{t}^{\{x, y, z\}}=\sum_{d=-D}^{D} d \cdot g_{t+d}^{\{x, y, z\}} / \sum_{d=-D}^{D} d^{2}$, where the shift $D$ is parameterized to the algorithms and $g_{t}^{\{x, y, z\}}$ stands for each of the three axis $\mathrm{G}$ components.

7. The Shifted Delta Coefficients (SDC) for estimating the first order time derivative of the BA signal components in the vicinity of the current timestamp [12]: $\Delta b_{t+i \cdot P}^{\{x, y, z\}}=$ $\frac{\sum_{d=-D}^{D} d \cdot b_{t+i-P+d}^{\{x, y)}}{\sum_{d=-D}^{D} d^{2}}$, where $b_{t}^{\{x, y, z\}}$ stands for each one of the three axis BA components, $N$ is the number of base features from which they are calculated, $D$ stands for the same as in the delta calculations, $P$ is the distance between samples and $K$ is the number of samples taken.

8. The Average Energy (AE) $[13,9,7]$ calculated as the sum of the squared discrete FFT component magnitudes of the signal in a window of a fixed size. This features allows to discriminate between static and dynamic activities. Although it is calculated for each axis, the aggregation or the average over the three axes is often used [7].

9. The correlation between axes [13] calculated for each pair of axes as the ratio of the covariance and the product of the standard deviations. This feature is useful to discriminate one dimensional activities if the sensor is properly placed. As stated in [7], this feature allows the discrimination between walking and climbing stairs.

10. The Intensity of the Movement (InMo) [14], which is the mean first derivative of the raw acceleration data, $\operatorname{InMo} o_{t}^{v \in\{x, y, z\}}=$ $\frac{1}{w} \sum_{i=0}^{w-1} \cdot\left|a_{t-i}^{v}-a_{t-i-1}^{v}\right| / \Delta x_{t} . \Delta x_{t}$ represents the time between samples, which can be ignored if the sampling rate is kept constant. The window size is given by the value of $w$.

11. The Time Between Peaks (TBP) [8], time in milliseconds between peaks in the sinusoidal waves associated with the frequency response of most activities (for each axis).

12. The Binned Distribution $[7,8]$ as stated by the authors, this measure is used with sliding windows of size $w$. For each window the range should be calculated as maximum-minimium; then, the range is divided into 10 equal size bins; finally, it is recorded what fraction of the $w$ values falls within each of the bins. This approach is named as Relative Binned Distribution (RBD). In this study, it is proposed the Absolute Binned Distribution (ABD) that is calculated using the lower and upper acceleration values as the range to be divided in bins.

In many of the solutions, sliding windows (with or without shifting) are proposed and the typical window size converges to the samples within a period of $2 \mathrm{~s}$. Features are typically normalized to 0 -mean 1-standard deviation and/or scaled to the interval 
$[0,1]$ before further preprocessing. Using frequency-derived features, employing FFT or similar ones, over long time-windows have been found more suitable for long duration and quasiperiodic signals like walking, cycling or teeth brushing. Otherwise, when classifying shorter duration and non-periodic activities, transitions or a short sequence of steps, then the time-domain representation has been found as a better solution [12].

\subsection{Previous work on $H A R$}

The characterization of human movement, specially while walking, is well documented in the literature [1]. Nevertheless, disruptive events, such as injury falling, strongly modifies the way patients move [15]. Particularly the patient's gait is severely affected. Due to this reason, and also because walking is one of the most sensible activities in human dependence, the gait and the patient kinematics have been broadly studied in the literature so far $[15,16]$.

Most of the previous studies are based on the analysis of videoimages of the patient gait or movements through well-known mechanical methods [16-19]. These studies are mainly focused on the rehabilitation of the patient and aimed at developing new therapeutical training techniques, providing interesting conclusions, and determining the relevant variables for characterizing the gait pattern.

Since the appearance of low cost and high performance accelerometers in the market, the recognition of human activity got the focus. Plenty of studies have analyzed the performance of this type of devices for stroke rehabilitation evaluation [20] and activity level measuring [21]. Some of the studies reported the used of different sensors and techniques, like accelerometers and electromyography [22] or accelerometers and electrocardiograph sensors [3]. One of those studies presented the combination of accelerometers and pressure gauges within the shoes for discriminating between three activities (sitting, standing and walking) of stroke patients [23]. In this study, Support Vector Machines (SVM) were proposed for classification of the activity, and rates of $99 \%$ of recall and $76.9 \%$ of precision were achieved. Though the classification performance is relatively good, it is worth noting that this approach can only discriminate between normal and abnormal walking as the rest of the activities are mainly related to the upper limbs.

There are also several studies concerning the use of accelerometers as the only source of information for HAR. [24] was one of the very first studies in HAR using accelerometers, in which several feature extraction methods were applied before modeling the classifiers of the different activities. Three different classifiers were applied: for the raw accelerometer data from the two accelerometers - one on each hip - for the subsets of Principal Component Analysis (PCA) and Independent Component Analysis. In each case, the most relevant feature subset were normalized to 0-mean and 1-standard deviation; an sliding window of 256 samples with 64 sample shift was used. Afterwards, wavelet transformations were carried out over the windowed data. A Multi-Layer Perceptron was trained with back propagation for the classification of four different activities: Stop, Walking, Walking Upstairs, Walking Downstairs. The classification error was used for evaluation each model, and the back propagation weight updated was based in the momentum.

A well known contribution to the field of HAR was proposed in [25]. In that study, the authors proposed the use of the divide and conquer strategy, detecting first static postures from dynamic activities. Then, specific decision trees were generated for each case, either static or dynamic. The framework was structured around a binary decision tree in which movements were divided into classes and subclasses at different hierarchical levels. General distinctions between movements were applied in the top levels, and successively more detailed subclassifications were made in the lower levels of the tree. This framework was used to develop a classifier to identify basic movements from the signals obtained from a single, waist-mounted triaxial accelerometer. Nevertheless, the main drawback of these methods is the computational complexity of the calculations that prevents them from being deployed in embedded devices.

An extension of the former study was presented in [26], where a sensor in the hip was used. The movements were first divided into activity (dynamic activities) and rest (static activities) using the SMA. Then, according to the postural orientation, it was proposed to decide the current activity and orientation. In spite of the obtained results from this approach and its low complexity, the main drawback of this study is that apart from falling, no other abnormal behavior can be detected using the sensor in the hip.

In [12] a rule and heuristic-based decision system is proposed for discrimination between the states of lying, standing, walking, as well as with the transitions between states. The transitions rules are learned through a Gaussian mixture model. This work is very interesting in the sense that it keeps track of the current state, that is, the cHA. Nevertheless, as far as only one sensor in the hip is used, this is not valid for the problem faced in present study. Moreover, the tilt of the body as well as the orientation of the body are rather different for present study.

In [2], Hidden Markov Models are proposed for automatic segmentation and classification of HAR. The underlying idea is that determining the activity that the current TS belongs to is no longer needed; only the number of activities to be identified are needed. To show the results up to 5 sensors, 4 triaxial accelerometers and one gyroscope were distributed among the chest, upper arms, ankle and thigh, respectively. The promising results in auto segmentation lacks in requiring a relatively high number of sensors. Similarly, a genetic algorithm driving the learning of a Fuzzy Finite State Machine was proposed in [6], using a sensor placed in the central part of the body. This study, which was called GFFSM, was found very suitable and will be further explained in the next subsection.

A very interesting idea is shown in [4], where a sparse representation of the input feature domain is proposed. This representation uses TS windows as a set of relevant motifs for each activity, each motif is the input feature with the information from the TS window. Whenever a new TS window is to be classified, the most suitable set of motifs is determined and thus the corresponding activity is proposed. Though this approach is conceptually rather novel and interesting, the main drawback of this method is again the computational cost that makes this method unfeasible for being introduced in embedded devices.

The use of triaxial accelerometers on the wrist is documented in $[10,7]$. In the former approach, up to 24 features were analyzed using dynamic Linear Discriminant Analysis; the best ranked features in each step drive the basis function classifier update to an iterative process that ends when a suitable error rate is obtained. Interestingly, this approach allows to evolve the activity set to detect, though the activity set applied in the experimentation is not described. The study presented in [7] details the general procedure for obtaining the set of features and, in this case, the neural classifier. Interestingly, this study proposed the common PCA for choosing the best feature subset, though they proposed the feature extraction instead of FS.

Currently, there is a trend to introduce HAR as an add-on to smartphones as far as the deployment cost is highly reduced $[14,8,27]$. In [14], three sensors were placed in the dominant wrist, hip and ankle; up to six different activities were studied: resting, typing, gesticulating, walking, running, and cycling. To classify the activity, two well known methods were analyzed: a C4.5 tree and a Feed-forward Neural Network, although only the latter was found useful. Similarly, but with a different feature subset, [8] proposed 
the time between peaks and a discretization of the sliding window to feed a J48 decision tree. Finally, a discussion about the benefits of using the data from sensors included in smartphones is presented in [27].

Present study focuses on choosing the best feature subset for a specific HAR model. The FS is a two-step method, an initial filtering one followed by a wrapper FS algorithm. After that, the final HAR model is learned.

\section{Proposed soft computing method for HAR}

This section introduces the lightweight HAR method proposed in this study to differentiate a reduced set of activities. To do so, two small wearable sensors are placed on the wrists of the person under analysis, as previously described. Once these data are gathered, a two-stage method is applied, as depicted in Fig. 1.

The well-known GFFSM HAR method is applied in present study due to its easy implementation in embedded devices. Nevertheless, this method must be adapted to work in unison with the sensors on the wrists because the original approach features does not reflects the current sensor placement. In addition, the problem of choosing the most suitable raw acceleration transformations is still present with the aforementioned adaptation. Consequently, using sensors located on the wrists introduces the problem of selecting which are the most suitable data transformations for the GFFSM performance. Therefore, the idea underneath is based on reducing the dimensionality of the input domain by means of a two-stage FS, a former filtering stage based on ICC and a latter stage using a wrapper FS algorithm.

As it is known, a wrapper FS method chooses the feature subset with the best fitness value. To evaluate each feature subset, the FS method learns a certain classifier or model and the chosen error measure is assigned as the fitness value of the feature subset. Thus, when the learning is complete, a wrapper FS method produces the feature subset and the model itself. Nevertheless, in most cases, the learning of the model during the feature selection is relaxed to avoid high computational costs. Therefore, once finished and the best feature subset is chosen, then the process ends with a full learning of the model.

In this study it is adapted a well-known hybridized method: the Steady-state Genetic Algorithm (SSGA) [28]. In this method, a Genetic Algorithm (GA) evolves the feature subset while, for evaluating the feature subset, a GFFSM is learnt for the feature subset with a relaxed set of parameters. This GA is a steady-state approach with a percentage of elite individuals defined a-priori. After the FS, the GFFSM is learnt with the complete parameter set for the best feature subset found so far. The whole approach is presented in Fig. 1.

Up to 150 features can be generated by the different transformations introduced in Section 2.1. Due to this huge amount of features, using the wrapper FS previously detailed would lead to high computational costs. Therefore, a filtering FS is deployed to reduce the dimensionality to the 20 most promising features before the wrapper FS is applied. This former stage will evaluate each feature with the Information Correlation Coefficient (ICC), and the most interesting features will be selected for the wrapper FS stage.

In the following subsections the stages of the proposed method are comprehensively described. Firstly, the GFFSM stage is presented and its adaptation is detailed. Then, the filtering and wrapper FS stages are described.

\subsection{Genetic fuzzy finite state machine stage}

In this study, the solution proposed by [6] is applied; consequently, this subsection is devoted to briefly describing this

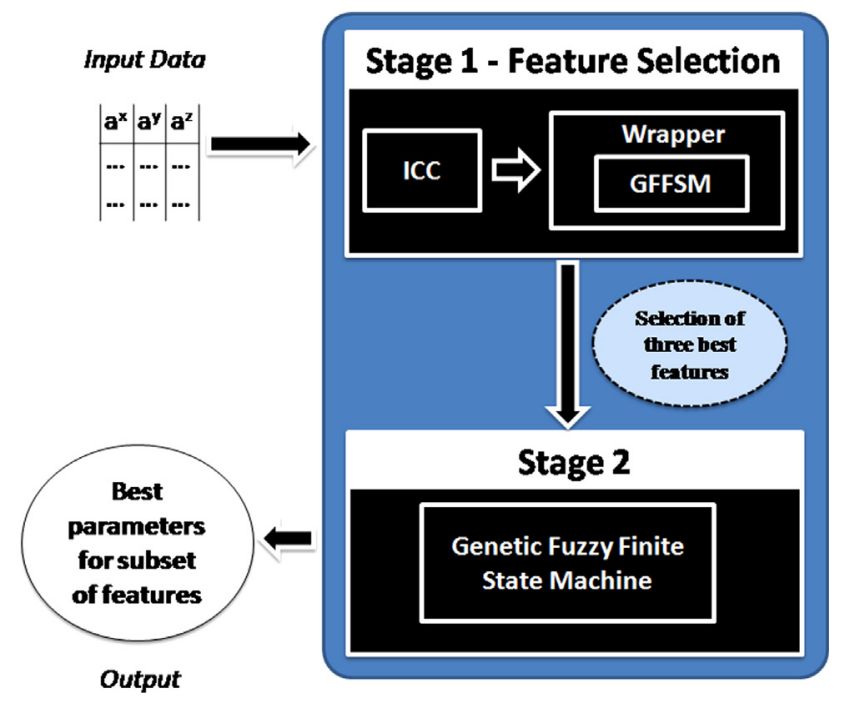

Fig. 1. The two-step FS method. After data gathering and pre-processing, the input feature domain is calculated. The FS comprises an ICC step where the most representative features are determined and a GFFSM-based wrapper-like FS method. The final GFFSM model is obtained afterwards, using the best feature subset.

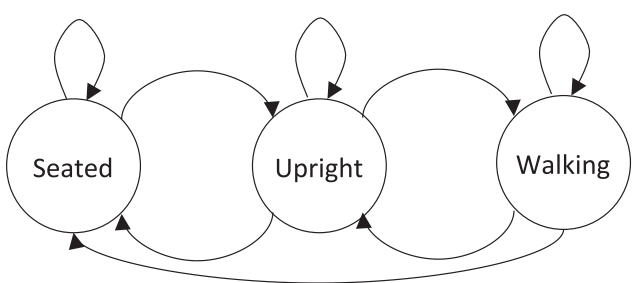

Fig. 2. The Fuzzy Finite State Machine proposed for this study. The allowed transitions defined the structure and consequents of the fuzzy rules, while the final antecedents are tuned with the learning process.

approach. The main contribution presented in [6] is a GA driving a Pittsburg approach of the Fuzzy Finite State Machine for detecting human activity $G F F S M=\{Q, U, f, Y, g\}$, running the rules and learning the partitions. The rules to be tuned are extracted from a predefined Finite State Machine, which is depicted in Fig. 2, considering only a set of three states \{Seated, Upright, Walking\}.

This approach makes use of one triaxial accelerometer placed in the center of the back, and three input variables are used: the dorso-ventral raw acceleration, the amount of movement and the tilt of the body. For each input variable, three linguistic labels $\left(n_{i}=3, \forall i\right)$ with Ruspini trapezoid membership functions are used, those $n_{i}+1$ parameters are needed to be learnt for each input variable. A GA evolves the partitions and the rules in a Pittsburg style as 72 binary genes encode part for the rules; 12 real-coded genes for the membership function parameters. The BLX- $\alpha$ crossover operator was proposed as focused on only one antecedent of chosen rules.

The fitness function is the mean absolute error (MAE), calculated as $M A E=\frac{1}{N} \frac{1}{T} \sum_{i=1}^{N} \sum_{j=0}^{T} a b s\left(s_{i}[j]-S_{i}^{*}[j]\right)$, where $T$ is the number of examples in the data set, $s_{i}[t]$ and $s_{i}^{*}[t]$ are the degree of activation and the expected degree of activation, respectively, of state $q_{i}$ at time $t=j$. The GA is completely defined with a binary tournament, generational replacement with elitism, two-point crossover for the rule base and BLX- $\alpha$ crossover for the realcoded genes that is applied twice for obtaining two new pair of chromosomes and classical bitwise mutation for the rule base. Additionally, uniform mutation is applied for the real-coded part. Termination occurs whenever the first of the following conditions 
applies: MAE takes 0 value, the expected number of generations is reached or there are a number of generations without changes in MAE. The remaining genetic parameters are: 100 individuals and 300 generations.

Nevertheless, this approach can not be directly applied in the current study as far as the sensors are placed on the wrists; therefore, the amount of movement, the dorso-ventral acceleration, and the tilt of the body, though easy to compute, looses its meaning.

\subsection{Filtering feature selection stage}

Filtering is usually employed when a big amount of features are available and there is a suitable measurement for ranking them. Nevertheless, when talking about HAR, rather few researches have been reported with this scope. A preliminary study of the most interesting features using the ICC measurement over the whole data set was carried out in [29]. However, in this study, an analysis using the ICC as the ranking measurement is defined but for different cross validation (CV) schemes.

Firstly, a standard experiment should be defined, and several runs of this experiment should be done; each run will provide us with a time series (TS) data set.

Secondly, three different feature datasets are generated with the available TS data sets: (i) DATA1, a global one including all the data gathered, (ii) DATA2, a $5 \times 2 \mathrm{CV}$ style grouping of TS, and (iii) DATA3, a 10-fold CV style grouping of TS. Both DATA2 and DATA3 comprise up to 10 different data sets including data from different TS.

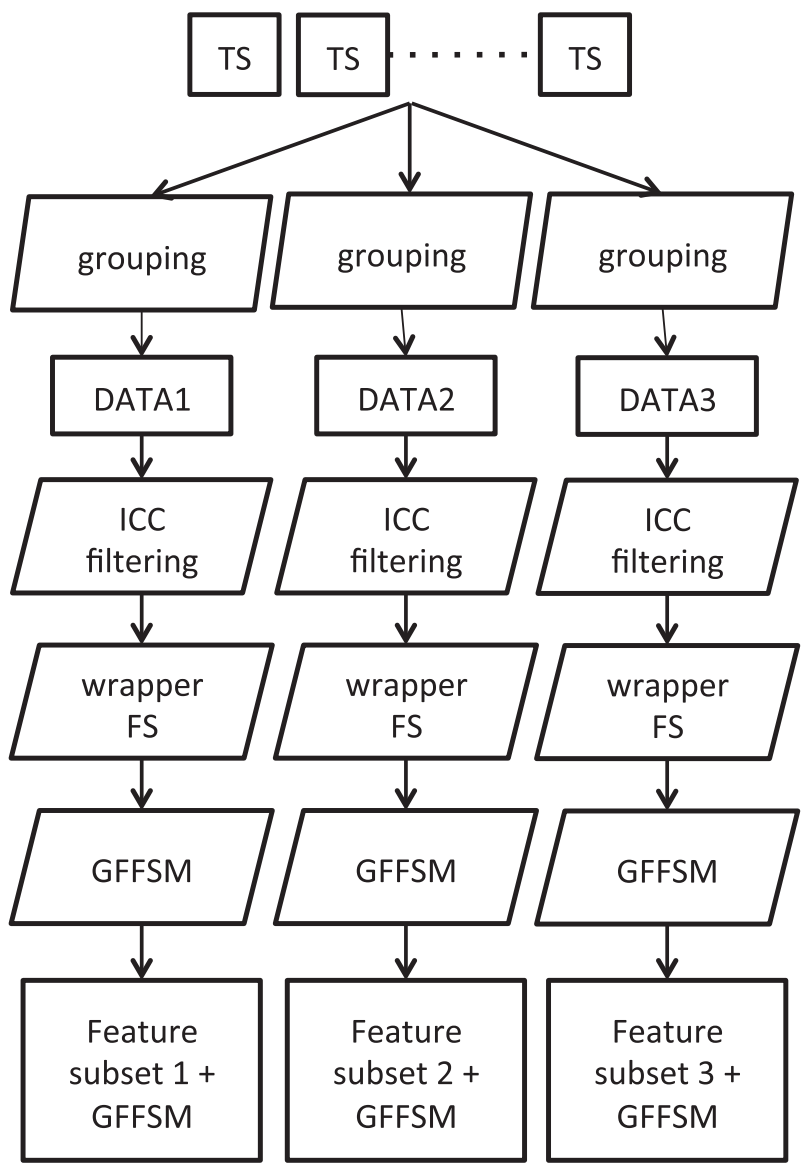

Fig. 3. The proposed FS procedure: the overall outcome includes a feature subset and the corresponding GFFSM model.
The main reason for these groupings is to evaluate how the ICC is biased due to the different schemes and thus, when high differences are found, it would mean that ICC is seriously biased and perhaps it might not be the best measurement for FS. In the case of DATA 1, the 20 best-ranked features are chosen for the wrapper FS. In the cases of DATA2 and DATA3, the 20 best-ranked features are selected from each fold and the union of all these folds generates the outcome for the next FS stage. Interestingly enough, the filtering stage will end up with three features subsets for further processing in the next FS stage.

\subsection{Wrapper feature selection stage}

The well-known SSGA FS method [30] is adapted to drive the learning of the GFFSM and also the selection of the best feature subset [29]. Thus, a GA will evolve the population of individuals, each individual representing a subset of three features from the input space. The parameters for the GA devoted to FS are 30 generations with 26 individuals, using the one-point crossover operator with probability 0.8 . The mutation operator is also flipping one of the three-selected features among the available candidates; the mutation probability is set to 0.02 . The fitness of each of the individuals is calculated as the MAE of the GFFSM model learnt from the feature subset the individual chooses.

Learning the GFFSM within the wrapper is relaxed to bound the computational costs: in this case, the GA runs 50 generations with 76 individuals, the $\alpha$-crossover operator probability is set to 0.8 for an $\alpha$ value of 0.3 . The mutation operator is the classical bitwise mutation for the rule base and the uniform mutation for the realcoded part; the mutation probability is set to 0.02 .

The following GA early stop conditions are defined: (i) the convergence measured as 25 generations without changes in the MAE of the best individual, and (ii) reaching a MAE fitness lower than 0.02 at any generation.

Finally, the GFFSM is obtained for each of the three different feature subsets, one for each case: DATA1, DATA2, and DATA3. The value for the GFFSM parameter subset is that proposed in the original research: 100 individuals, 300 generations and the same crossover and mutation operators and probabilities. This final GFFSM model is referred for comparison purposes as WRAPPER Fig. 3 illustrates the whole feature selection stage and its outcome.

\section{Evaluation and experimentation}

As previously stated, this study focuses on early stroke diagnosing, thus the HAR analysis is always concerned with the typical activities of the focused population, namely WALKING, STANDING and RESTING. The experimental validation performed in this study is aimed at evaluating which one of the proposed alternatives is the best for the recognition of these three activities. To do so, three different datasets have been generated and analyzed. The obtained results are described in this section.

In order to gather the data, the subject under analysis is provided with two bracelets Fig. 4(b) each one of them with a triaxial accelerometer - the ADXL345 sensor has been used in present study - with a sampling frequency of $16 \mathrm{~Hz}$. Ten runs of a typical rehabilitation test Fig. 4(a) will be carried out and all the data will be manually segmented and classified according to the activity the subject is owe to do. This segmentation is performed in a similar way to that stated in the original GFFSM study [6]: known states are assigned with total certainty, while transitions are assigned with imprecise observations, e.g., 0.7/SEATED +0.3/ STANDING.

To obtain results from the bracelets the analyzed subject stayed seated for a period of time T1, then stood up and stayed in that 
a

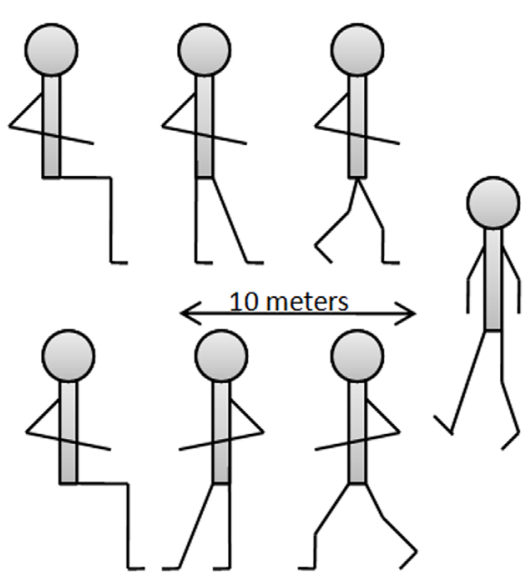

b

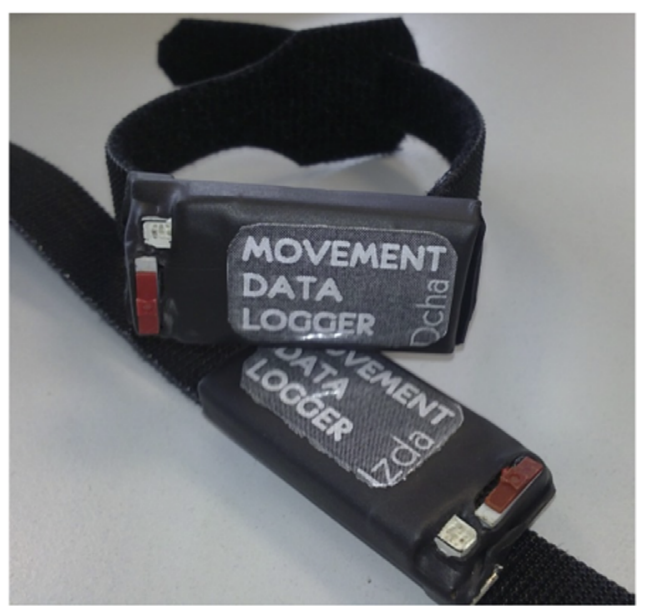

Fig. 4. (a) The rehabilitation test carried out with different subjects movements. (b) The set of bracelets used in this experimentation, one for each wrist.

position for a period of time $\mathrm{T} 2$, then started walking following a $10 \mathrm{~m}$ straight line on the floor and went back to the original standing up location, stayed stand up for T2 seconds and finally seated down and stayed resting for T1.

The rehabilitation test carried out involved the following steps (see right side of Fig. 3): the subject should be seated during a time T1, then should stand up and stay on that position during T2. Afterwards, the subject will start walking following a straight line on the floor $(10 \mathrm{~m})$ and go back. The subject shall stand up during T2 and, finally, keep seated during T1. T1 and T2 have been set to $60 \mathrm{~s}$.

The experimentation, thus, will include (i) the data gathering as explained before, (ii) computing the transformations, (iii) performing the FS: the former filtering step followed by the wrapper FS with the relaxed GFFSM, (iv) learning the adapted GFFSM as explained in Section 3.1, and (v) learning the GFFSM with the best feature subset and with the complete GFFSM parameter set. A flowchart of the experimentation for each of the arrangements (DATA1, DATA2 and DATA3) is depicted in Fig. 5.

Two cross validation schemes $(5 \times 2$ and $10 \mathrm{f})$ are used to study how the features help in each case for obtaining a suitable model. In case that for certain data arrangement the best model is found for both CV schemes, it can be said that arrangement is clearly the best one. Next subsections deliver the results obtained from each one of the stages: FS and GFFSM learning.

\subsection{Feature selection results}

As stated above, the DATA1 processing generates a reduced space containing the 20 best-ranked features. For the remaining arrangements, it would depend of the features chosen from each fold. In this case, 22 and 31 features were finally chosen from DATA2 and DATA3, correspondingly (Fig. 6).

The feature subset obtained for each arrangement after the filter and the wrapper FS steps are depicted in Table 1.

\subsection{GFFSM alternatives for HAR modeling}

Once the best feature subset for each arrangement has been found, its time for modeling. Interested reader should recall that for each feature subset obtained from FS on one of the data arrangements - a GFFSM model is to be obtained using a combination of window size and cross validation as shown in Fig. 5.

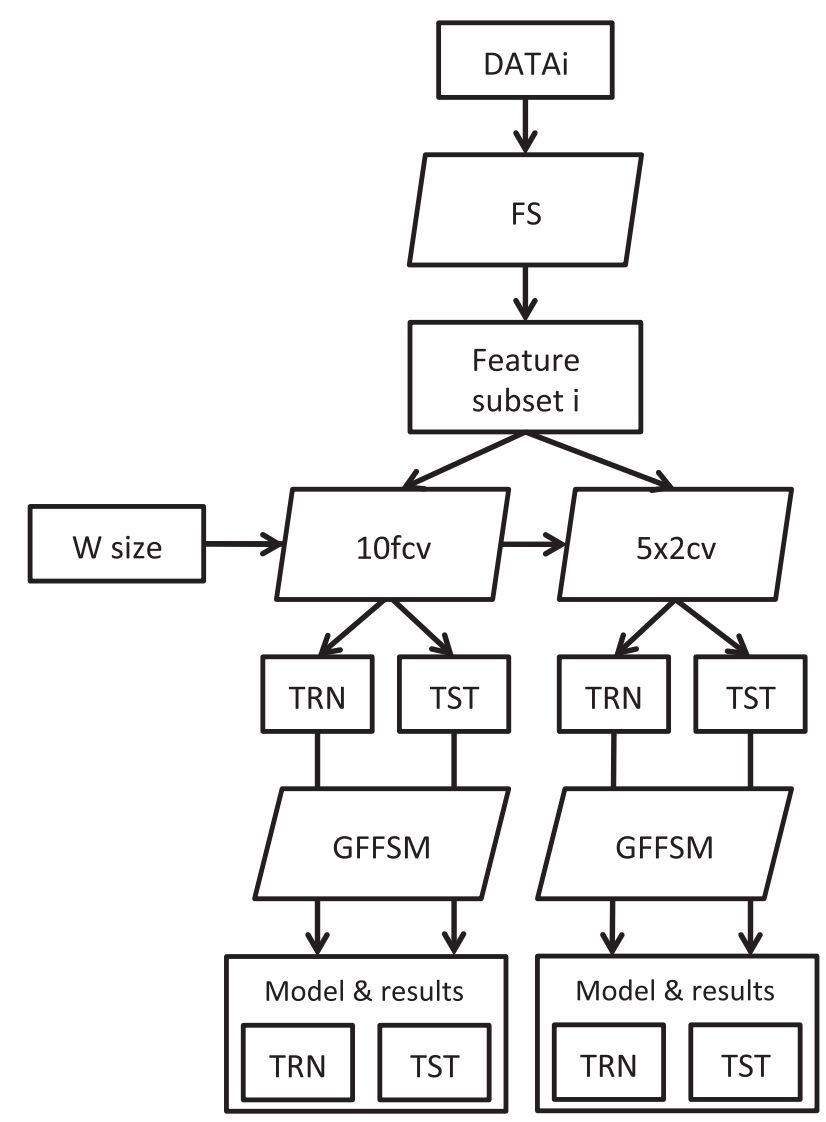

Fig. 5. Flowchart of the experimentation for each DATA arrangement. Once prepared the DATAi, FS takes place outcoming with the feature subset. Consequently, the original data set for these features is prepared and two cross validation schemes are employed: $5 \times 2$ and $10 \mathrm{f} \mathrm{CV}$. For each fold, a GFFSM is learnt and results from training and testing are obtained. TRN and TST stands for the corresponding folds used in training and test; consequently, with the TRN/TST data sets the train/test results are obtained. These folds are generated according to the cross validation scheme.

Table 2 includes the results for all the possible combinations, while the boxplots with the MAE for the best individual obtained for each fold can be seen in Fig. 7, for a window size of 10 samples.

The feature subset obtained from the arrangement DATA3 performs better in both $5 \times 2$ and $10 \mathrm{f} \mathrm{CV}$. Therefore, it can be 


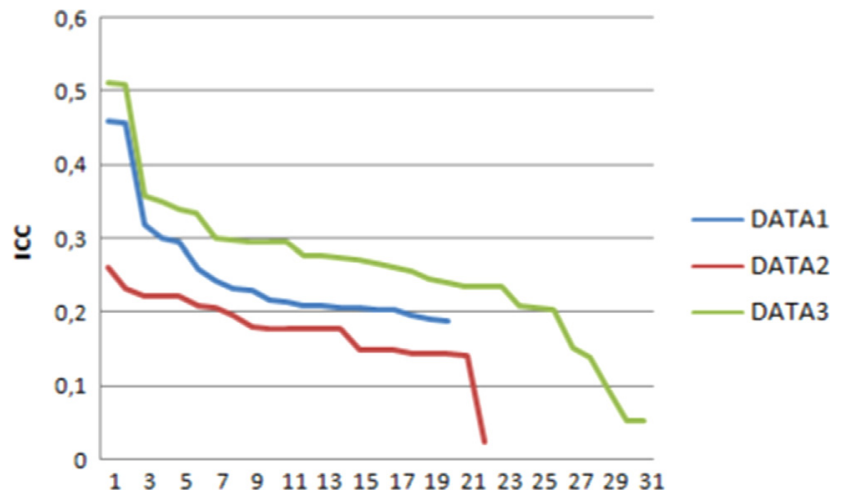

Fig. 6. For DATA1 the results of the 20 ICC best features. For the DATA2 and DATA3 the average of the ICC results in the 10 folders.

Table 1

Feature subsets for each arrangement after the two-step FS.

\begin{tabular}{lll}
\hline DATA1 & DATA2 & DATA3 \\
\hline$a^{x}$ & $a^{x}$ & $a^{x}$ \\
$g^{y}$ & $R M S^{y}$ & $R M S^{x}$ \\
$R B D^{y}$ bin $=10$ & $T B P^{y}$ & $A M$ \\
\hline
\end{tabular}

Table 2

Statistical test results for the MAE. Std stands for standard deviation.

\begin{tabular}{|c|c|c|c|c|c|c|}
\hline \multirow[t]{2}{*}{ Measure } & \multicolumn{2}{|c|}{ Feature subset 1} & \multicolumn{2}{|c|}{ Feature subset 2} & \multicolumn{2}{|c|}{ Feature subset 3} \\
\hline & $5 \times 2$ & 10 K-Folder & $5 \times 2$ & 10 K-Folder & $5 \times 2$ & 10 K-Folder \\
\hline Average & 0.0509 & 0.0408 & 0.0490 & 0.0418 & 0.0332 & 0.0199 \\
\hline Median & 0.0400 & 0.0391 & 0.0391 & 0.0369 & 0.0209 & 0.0208 \\
\hline Deviation & 0.0298 & 0.0134 & 0.0276 & 0.0197 & 0.0306 & 0.0041 \\
\hline
\end{tabular}

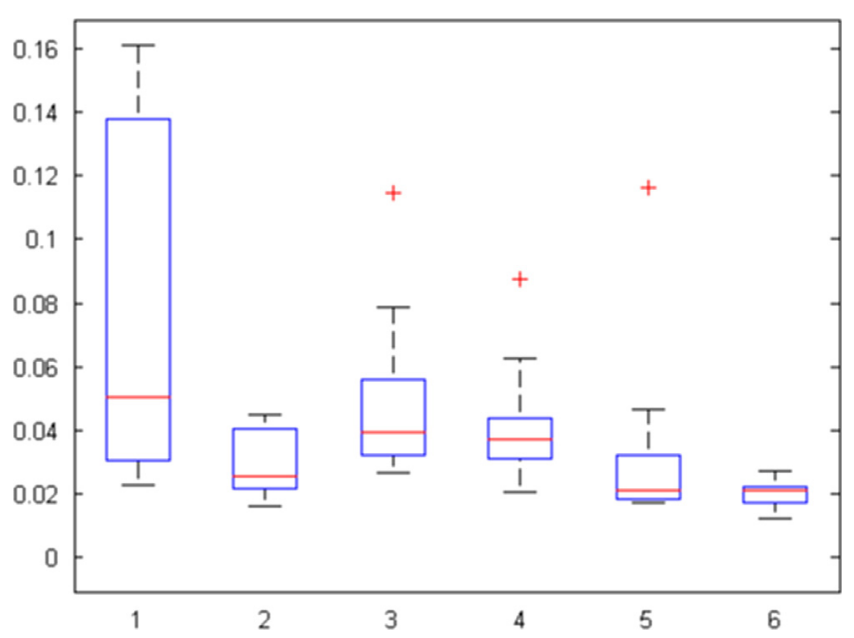

Fig. 7. Features subset 1 for $5 \times 2$ and $10 \mathrm{~K}$-Folder CV, Features subset 2 for $5 \times 2$ and $10 \mathrm{~K}$-Folder CV and Features subset 3 for $5 \times 2$ and $10 \mathrm{~K}$-Folder CV are marked on the boxplot, respectively.

Table 3

MAE testing rates for the GFFSM-a [11] (with features SMA, $\Delta$, and the AM), the GFFSM-w [29] (with features $a^{x}, g^{y}$, and $R B D^{y}$; bin $=10$ ), and the Wrapper (with features $a^{x}, R M S^{x}$, and $\left.A M\right)$.

\begin{tabular}{llll}
\hline Measure & GFFSM-a & GFFSM-w & WRAPPER \\
\hline Average & 0.0391 & 0.0365 & 0.0199 \\
Median & 0.0306 & 0.0343 & 0.0208 \\
Deviation & 0.0292 & 0.0098 & 0.0041 \\
\hline
\end{tabular}

concluded that this feature subset is the best candidate for the final application.

In Table 3, the statistical results for the three different GFFSM releases are depicted. The model obtained after the FS stage really outperforms the rest of candidates; consequently, the FS selection stage and the subsequence analysis is found valid. With these results we can conclude that the HAR recognition stage for an early stroke diagnosis tool is not conclude but satisfactory, thus its time to focus on alarm generation. Further HAR studies should be focused on learning the GFFSM rules as well as the partitions, which might reduce the number of rules and also the computational resources needed for integrating these models in embedded devices.

\section{Conclusions}

This study presents a Soft Computing method for modeling and validation in HAR, focusing on three different activities: being seated, standing up and walking. The main objective of this study is choosing the best combination of parameters to get the subset of features that best describes this HAR problem while the activity conditions are modeled at the same time. So, this study proposes a FS method to reduce the space dimensionality of features that differs from previous work.

In keeping with this idea, a Hybrid Artificial Intelligent Method has been designed an applied. In a first stage, a filter FS is deployed to reduce the dimensionality by evaluating each feature with ICC. Then, the most important features are selected for the wrapper stage. Since the features are chosen, a wrapper FS method using GFFSM is the responsible of searching for the best feature subset associated with each data set and for modeling the human activity. Finally, different schemes are used for validation of the best parameters for features subset and the model from the best data set with different runs.

From the obtained results some conclusions are drawn. Firstly, the use of a cross validation scheme for feature selection might improve the overall outcome. Perhaps a voting scheme may also improve the performance of the feature filtering stage. Secondly, the window size has been found problem specific, thus the different studies in the literature should have made such analysis without reporting it. Finally, it has been found a proper feature subset and the corresponding GFFSM model for HAR, which is to be deployed in further research concerning early stroke diagnosis.

Future work envisages the implementation of more stages in the Finite State Machine, easing the experts reutilization of the presented approach. Further research would be done to include others styles to tackle the learning for the fuzzy rule base of the GFFSM instead of predefining it.

\section{Acknowledgments}

This research has been funded by the following grants: Spanish Ministry of Science and Innovation (TIN2011-24302 and PID 560300-2009-11 projects), Fundación Universidad de Oviedo (FUO-EM-340-13 project), Junta de Castilla y León-SACYL (BIO/ BU09/14), Castilla y León Health Care System project SACYL 2013 (GRS/822/A/13).

\section{References}

[1] M.P. Murray, A.B. Drought, R.C. Kory, Walking patterns of normal men, J. Bone Jt. Surg. 46 (2) (1964) 335-360.

[2] F. Chamroukhi, S. Mohammed, D. Trabelsi, L. Oukhellou, Y. Amirat, Joint segmentation of multivariate time series with hidden process regression for human activity recognition, Neurocomputing 120 (23) (2013) 633-644. 
[3] T. Fujimoto, H. Nakajima, N. Tsuchiya, H. Marukawa, K. Kuramoto, S. Kobashi, Y. Hata, Wearable human activity recognition by electrocardiograph and accelerometer, in: IEEE 43rd International Symposium on Multiple-Valued Logic, 2013.

[4] M. Zhang, A.A. Sawchuk, Human daily activity recognition with sparse representation using wearable sensors, IEEE J. Biomed. Health Inform. 17 (3) (2013) 553-560

[5] J. Sedano, S. González, B. Baruque, Á Herrero, E. Corchado, Soft computing models in industrial and environmental applications, in: V. Snásel, A. Abraham, E.S. Corchado (Eds.), Soft Computing for the Analysis of People Movement Classification of Advances in Intelligent Systems and Computing, 188, Springer, Berlin, Heidelberg, 2013, pp. 241-248.

[6] A. Ávarez-Álvarez, G. Triviño, O. Cordón, Body posture recognition by means of a genetic fuzzy finite state machine, in: IEEE 5th International Workshop on Genetic and Evolutionary Fuzzy Systems (GEFS), 2011, pp. 60-65.

[7] J.-Y. Yang, J.-S. Wang, Y.-P. Chen, Using acceleration measurements for activity recognition: an effective learning algorithm for constructing neural networks, Pattern Recognit. Lett. 29 (2008) 2213-2220.

[8] J.R. Kwapisz, G.M. Weiss, S.A. Moore, Activity recognition using cell phone accelerometers, ACM SIGKDD Explor. Newsl. 12 (2) (2010) 74-82.

[9] S. Wang, J. Yang, N. Chen, X. Chen, Q. Zhang, Human activity recognition with user-free accelerometers in the sensor networks, in: Proceedings of the International Conference on Neural Networks and Brain ICNN \& B'05, vol. 2, IEEE Conference Publications, Beijing, 2005, pp. 1212-1217.

[10] Y.-P. Chen, J.-Y. Yang, S.-N. Liou, G.-Y. Lee, J.-S. Wang, Online classifier construction algorithm for human activity detection using a triaxial accelerometer, Appl. Math. Comput. 205 (2) (2008) 849-860.

[11] S. González, J.R. Villar, J. Sedano, C. Chira, A preliminary study on early diagnosis of illnesses based on activity disturbances, in: S. Omatu, J. Neves, J.M.C. Rodríguez, J.F. Paz Santana, S.R. Gonzalez (Eds.), Distributed Computing and Artificial Intelligence Advances in Intelligent Systems and Computing, vol. 217, Springer, Berlin, Heidelberg, 2013, pp. 521-527.

[12] F.R. Allen, E. Ambikairajah, N.H. Lovell, B.G. Celler, Classification of a known sequence of motions and postures from accelerometry data using adapted Gaussian mixture models, Physiol. Meas. 27 (2006) 935-951.

[13] L. Bao, S.S. Intille, Activity recognition from user-annotated acceleration data in: A. Ferscha, F. Mattern (Eds.), Proceedings of the Second International Conference Pervasive Computing, PERVASIVE 2004, Lecture Notes in Computer Science, vol. 3001, Springer, Berlin Heidelberg, 2004, pp. 1-17.

[14] N. Györbiro, Á Fábián, G. Hományi, An activity recognition system for mobile phones, Mob. Netw. Appl. 14 (2009) 82-91.

[15] M. Ahmed, S. Ahmed, Kinetics and kinematics of loading response in stroke patients (a review article), Ann. King Edward Med. Univ. 14 (4) (2008) 143-147.

[16] I. de Quervain, S. Simon, S. Leurgans, W. Pease, D. McAllister, Gait pattern in the early recovery period after stroke, J. Bone Jt. Surg. Am. 78 (10) (1996) 1506-1514.

[17] H.P. von Schroeder, R.D. Coutts, P.D. Lyden, E Billings Jr., V.L. Nickel, Gait parameters following stroke: a practical assessment, J. Rehabil. Res. Dev. 32 (1) (1995) 25-31

[18] K. Hollands, Whole body coordination during turning while walking in stroke survivors (Ph.D. thesis), School of Health and Population Sciences, University of Birmingham, 2010.

[19] S.-R. Ke, H.L.U. Thuc, Y.-J. Lee, J.-N. Hwang, J.-H. Yoo, K.-H. Choi, A review on video-based human activity recognition, Computers 2 (2013) 88-131.

[20] B. Knorr, R. Hughes, D. Sherrill, J. Stein, M. Akay, P. Bonato, Quantitative measures of functional upper limb movement in persons after stroke, in Conference Proceedings of the Second International IEEE EMBS Conference on Neural Engineering, 2005, 2005, pp. 252-255.

[21] D. Rand, J.J. Eng, S.-S. Chang, P.-F. Tang, J.-S. Jeng, C. Hung, How active are people with stroke?: use of accelerometers to assess physical activity, Stroke 40 (2009) 163-168.

[22] S.H. Roy, M.S. Cheng, S.-S. Chang, J. Moore, G.D. Luca, S.H. Nawab, C.J.D. Luca, A combined semg and accelerometer system for monitoring functional activity in stroke, IEEE Trans. Neural Syst. Rehabil. Eng. 17 (6) (2009) 585-594.

[23] G.D. Fulk, E. Sazonov, Using sensors to measure activity in people with stroke, Top Stroke Rehabil. 18 (6) (2011) 746-757.

[24] T.J. Mantyla, J. Himberg, Recognizing human motion with multiple acceleration sensors, in: IEEE International Conference on Systems, Man and Cybernetics, vol. 3494, 2001, pp. 747-752.

[25] M. Mathie, B. Celler, N. Lovell, A. Coster, Classification of basic daily movements using a triaxial accelerometer, Med. Biol. Eng. Comput. 42 (5) (2004) 679-687.

[26] D.M. Karantonis, M.R. Narayanan, M. Mathie, N.H. Lovell, B.G. Celler, Implementation of a real-time human movement classifier using a triaxial accelerometer for ambulatory monitoring, IEEE Trans. Inf. Technol. Biomed. 10 (1) (2006) 156-167.

[27] L.G.M. de la Vega, S. Raghuraman, A. Balasubramanian, B. Prabhakaran, Exploring unconstrained mobile sensor based human activity recognition, in: the Third International Workshop on Mobile Sensing, 2013.

[28] J. Casillas, O. Cordón, M. del Jesus, F. Herrera, Genetic feature selection in a fuzzy rule-based classification system learning process, Inf. Sci. 136 (1-4) (2001) 135-157.

[29] J.R. Villar, S. González, J. Sedano, C. Chira, J.M. Trejo, Human activity recognition and feature selection for stroke early diagnosis, in: the Eighth
International Conference on Hybrid Artificial Intelligence Systems HAIS, Lecture Notes in Artificial Intelligence, Springer, Berlin Heidelberg, 2013.

[30] J. Casillas, O. Cordon, M.J. Del Jesus, F. Herrera, Genetic feature selection in a fuzzy rule-based classification system learning process for high-dimensional problems, Inf. Sci. 136 (1-4) (2001) 135-157.

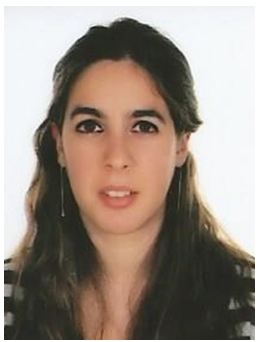

Silvia González obtained her Computer Science Engineering Degree at the University of Burgos in 2011. Since 2010 she is working with the Artificial Intelligence and Applied Electronics group at the Instituto Tecnológico de Castilla y León. She is currently also engaged in a Ph.D. in the area of Computer Engineering at the University of Oviedo, Spain. Her research interests are focused in Genetic Algorithms, Feature Selection and Machine Learning.

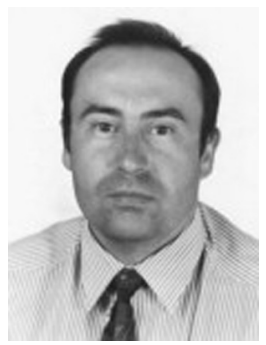

Javier Sedano is an expert in the development of electronic systems (hardware), industrial project, production-systems acquisition and control systems, as well as in the design of connectionless models for the identification and modelling of dynamic systems. He is the head of the Artificial Intelligence and Applied Electronics group at the Instituto Tecnológico de Castilla y León. He is also part of the Applied Computational Intelligence Group at the University of Burgos and has spent several years working on projects and publications related to artificial intelligence and system modelling. He has collaborated in the organization of international scientific conferences, program committees and organizations. $\mathrm{He}$ is a member of the IEEE Systems, Man \& Cybernetics Chapter Society Spanish Chapter and has several international publications as well as records of software and industrial patents.

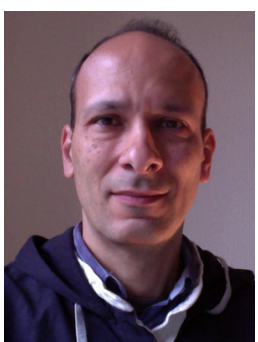

José R. Villar obtained his Electronic Engineering degree at University of Oviedo (1992) and his Ph.D. in Computer Science at University of León (2002). He has worked in several companies focused in different fields, from automation to electric power generation. He was a member of the Electronic and Engineering Department at University of León (1998-2004). Currently, he is an Associate Professor with the Computer Science Department at University of Oviedo.

His research topics are based on Genetic Fuzzy Systems and Soft Computing applied in real world systems. He has received public funds for his research in fields like Electrical Distribution Optimization and Bioinformatics. Moreover, he has signed research projects with local enterprises for developing new products or for research transfer; several patents have been approved as a consequence. Currently, he has published more than 20 research studies in indexed journals and more than 70 conference papers.

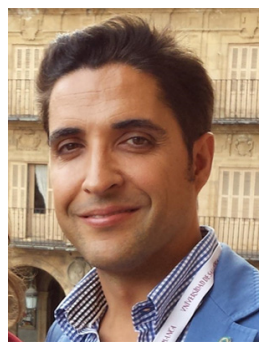

Emilio Corchado is Associate Professor at University of Salamanca and Full Professor accredited by ANECA since 15th February 2012. He is the President of IEEE Spain Section (2014-2015), founder and Vicepresident of Spanish Chapter SMC of IEEE. Founder of the GICAP Research Group of University of Burgos, member of IBSAL, and member of BISITE Research Group (University of Salamanca). He is the Coordinator of European Project WELCOME and partner of European project SEP, both projects under the initiative H2020 from European Commission.

He is president of 4 international congresses, he has been president of Organizing Board of 10 Congress/ Workshop of highly recognized prestige and member of Editorial Board of the following magazines: KAIS, IJNS, IJCIA, Computational Intelligence, IJRIS. He is/has been IP of more than 12 competitive national and regional projects of $\mathrm{I}+\mathrm{D}$, coordinator of 1 European project, member of another in H2020 framework, and member of 10 European competitive projects of $\mathrm{I}+\mathrm{D}$.

Besides he has been Coordinator of the node of University of Burgos and Salamanca of 2 European and 5 national thematic networks. He is member of several COST Actions (IntelliCIS, APP for AAL, etc.). He is author of more than 70 published articles in magazines with impact factor, 38 books and chapter of books of scientific character and author/coauthor of more than 120 published articles in international scientific congresses. 


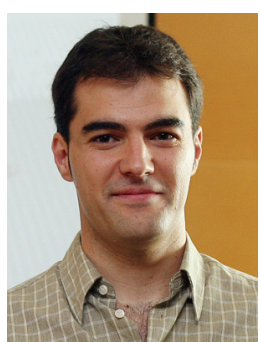

Álvaro Herrero is a lecturer in Artificial Intelligence at the Higher Polytechnic School of the University of Burgos (Spain). He has been an academic at Burgos since September 2005. Álvaro Herrero gained his PhD from the University of Burgos, where he researched hybrid artificial intelligence systems applied to network intrusion detection. His main research interests are related to applied artificial intelligence, including artificial neural networks (mainly those based on unsupervised learning), multiagent systems and hybrid artificial intelligence, specially addressing (but not restricted to) problems in the field of computer network security.

Álvaro Herrero has published a book, more than 10 papers in prestigious peer-reviewed journals and more than 60 papers in international conferences. He has served as a reviewer for several reputed international journals and has been a member of the international scientific committee of many international conferences such as ICANN, ISNN, IWANN, IDEAL, PAAMS, HAIS, IWANN, KES, CISIS, IAS, UCAmI, IWPACBB and DCAI among others. Álvaro Herrero has also been organizing chair, program committee chair, session chair and special session organizer for a number of conferences, such as CISIS, HAIS, IDEAL, SOCO, KES, ISDA and CMMSE.

Álvaro Herrero is the treasurer of the IEEE Systems, Man \& Cybernetics Society Spanish Chapter, and officer of the IEEE Spanish Section.

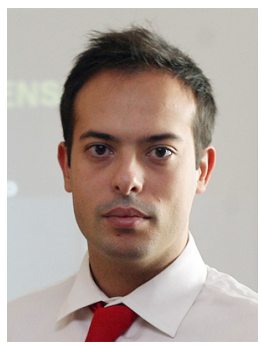

Bruno Baruque holds a lecturer position at the University of Burgos, Spain. He obtained his Ph.D. degree in Computer Science/Artificial Intelligence (European Mention) in 2009 and his Master Degree in Computer Science in 2004, both at the Univ. of Burgos.

He is an active member of the GICAP (Univ. of Burgos) and BISITE (Univ. of Salamanca) Research Groups, having participated in many of the projects that have been developed by those groups; both in the Computer Science Research and Education areas. He has also collaborated with other research groups at the LABRI (Bordeaux, France) or the University of Manchester (Manchester, UK), during research stays in the mentioned centres.
His research interests are focused on the automated learning field, with special emphasis in artificial neural networks. His work has mainly been related with unsupervised learning algorithms used for data visualization and ensemble learning models used to improve the results of those algorithms, having published many peerreviewed papers, in those topics including theoretical and practical applications (food industry, environmental analysis, etc.) in reputed international journals and conferences.

He collaborates as reviewer of several international journals (Neurocomputing Pattern Analysis and Applications, etc.) and as programme committee member in numerous conferences (Int. Conf. on Hybrid Artificial Intelligence Systems (HAIS), Int. Conf. on Soft Computing Models in Industrial and Environmental Applications (SOCO), Int. Conf. on Intelligent Data Engineering and Automated Learning IDEAL). 